\title{
Why are we seeing so many more children diagnosed with food allergies?
}

\section{Editorial}

With the alarming increase in food allergies, it is important to examine what might be going on. In fact there may be several things going on. The rise in childhood food allergies is rising in an almost parallel fashion to childhood obesity. Several decades ago (when I was in school, not to date myself), there were barely any children at all in school that had any food allergies. In addition, there were very few children who we would consider overweight. In fact, it wasn't until recently that we even had a diagnosis of childhood obesity. Now there are children who's weight percentile's are so high they can barely see the growth chart. We played outside, we sat down together to eat as families, and we ate out minimally. We still had packaged food (I'm not that old) but we ate them in smaller quantities and we ran around so we burned them off. But what does this have to do with food allergies? A lot!

When we are limited to indoor activities our bodies get inadequate amounts of vitamin D. If you live in the northern part of the country like I do, you do not synthesize vitamin D between the months of October and March. That's most of the year. And then when you are able to synthesize vitamin D, like the two months of summer that we get, you most likely are wearing sunscreen which blocks our skin's ability to synthesize vitamin D. So therefore most of us are vitamin D deficient, which leads to decreased ability to burn fat and an increase in our body's level of inflammation. What's more is increased fat cells cause vitamin D to be sequestered into the fat stores where they are not accessible so then we have decreased fat burning and increased inflammation. So what comes first, the obesity or the vitamin D deficiency? That answer is about as clear as whether the chicken or the egg came first. Where else can we can vitamin D? Milk! I personally don't like the taste of milk but if you do, 16 ounces a day can provide you the recommended amount of vitamin D. The calcium from milk can assist with our bodies ability to burn fat. Another source is shiitake mushrooms. To me that one is more desirable.

So, the vitamin $\mathrm{D}$, inflammation, obesity connection is one way in which obesity and food allergies are tied together. When our bodies are inflamed, our gastrointestinal track goes from being an intact tube to a pourous, holy vessel that allows translocation of allergens (as well as other molecules) to pass from through and introduce themselves to our immune system. Then the immune system develops a system where it then reacts to that molecule in the future. Those shiitake mushrooms as well as other mushrooms can help to decrease our bodies inflammation and help to ameliorate this cascade of events. Other foods that help to decrease our bodies inflammation are fish (limit to 12 ounces a week to decrease mercury exposure), avocado, walnuts, flax seeds (ground), chia seeds and hemp seeds. I start every day with a glass of water and one tablespoon of chia seeds mixed in.

How else is it connected? Many people who are overweight eat a diet low in fruits and vegetables and high in meats and other foods with a high fat content. This is known as an obesogenic diet. Not only are these diets higher in calories and therefore lead to an increase in weight gain, they are also lower in fiber and pre and probiotics. These worsens the problem of the porous gastrointestinal track, leading to increased translocation of allergenic foods. So eat more vegetables,
Volume 8 Issue 5 - 2018

\section{Theresa DeLorenzo}

Department of Nutrition and Human Performance, Logan University, USA

Correspondence: Theresa DeLorenzo, Program Director, Master of Science in Nutrition and Human Performance, Logan University, 23 Paddlewheel Court Waterford, NY 12188, USA, Tel (5I8) 429-7506, Email tharesadelorenzol23@yahoo.com

Received: October 17,2018 | Published: October 19, 2018

eat more fruit, eat more yogurt and green olives and decrease your consumption of meat and cheese. Do we have to eliminate foods and entire food groups to be healthy? NO! Please don't! Find the balance. Exercise. Get outside. Drink milk (or eat mushrooms).

Another major contributor is the increase in premature births. Premature infants are born before the accumulation of nutrients from mom and they are born before their gastrointestinal track has completely developed, among all of their other systems. In terms of allergy development the gastrointestinal development, it is the one that is most related. When the gastrointestinal is underdeveloped you can probably guess that it mimicks what happens when our gastrointestinal track is inflamed. It is pourous and allergens translocate and introduce them to the immune system. How is this related to obesity? Rates of prematurity are higher in the obese population. Infertility is higher in the obese population, which leads them to undergo fertility treatment which increases the odds of multiples who are typically born premature. Obesity also increases the odds of mom having preeclampsia, another contributing factor to prematurity. Multiples are more often delivered by c- section which decreases their exposure to the vaginal flora so they have a decreased ability to build their own flora. And if we hadn't mentioned enough reasons, prematurity is a huge stressor which makes breast feeding that much more difficult. Breast fed infants have decreased risk for obesity because they selfregulate their intake and they are exposed to a variety of flavors which lends them to a more varied diet as they get older, i.e. more fruits and vegetables than their non- breast fed counter parts. In addition, breast fed infants have decreased risk for developing allergies because of the oligosaccharides that breast milk provides. Another contributing factor to the development of allergies in the premature population. To take this a step further, premature infants and infants born to mom's who have pre- eclampsia have a metabolism that is often down regulated in utero to survive in an environment that is not providing them adequate nutrition. This lends them to be at higher risk for obesity. And the cycle continues.

\section{Acknowledgement}

None.

\section{Conflict of interest}

Author declares that there is no conflict of interest. 